\title{
An Adaptable and Personalised E-Learning System Based on Free Web Resources
}

\author{
Eiman Aeiad and Farid Meziane \\ School of Computing, Science and Engineering \\ University of Salford, Salford, M5 4WT, UK \\ E.Aeiad@edu.salford.ac.uk, f.meziane@salford.ac.uk
}

\begin{abstract}
A personalised and adaptive E-Learning system architecture is developed to provide a comprehensive learning environment for learners who cannot follow a conventional programme of study. The system extract information from freely available resources on the Web, and taking into consideration the learners' background and requirements to design modules and a planner system to facilitate the learning process. The process is supported by the development of an ontology to optimise the in-formation extraction process. An application in the computer science field is used to evaluate the proposed system based on the IEEE/ACM Computing curriculum.
\end{abstract}

Key words: E-Learning, Learning Styles, Personalized Learning

\section{Introduction and Motivation}

Learning is greatly influenced by the development of Information and Communication Technologies and advanced digital media. Learning using these new media is referred to as E-Learning [6]. It allows access to education to those who find it difficult to be physically present in the traditional classroom based learning. Together with the development of the Web, a plethora of freely available resources is accessible and used as part of E-Learning. Although, E-Learning may have addressed the needs of the masses, it failed to satisfy the needs of individual learners by providing them with personalised learning plans and resources.

In this paper, we present the Adaptive and Personalised E-Learning System APELS that extends the current understanding and use of conventional E-Learning resources, by using freely available resources on the Web to design and deliver content for individual learners. The system would be used by individual learners, universities that may not have the resources and the expertise to develop learning resources and anyone who wishes to learn a specific filed. At this stage, by learning we mean academic learning and by resources those available for academic learning. The APELS system will enable users to design their own learning material based on internationally recognised curricula and contents. Using standard search engines to find learning material that is suitable for individual learners is time consuming and may not lead to a suitable outcome. The major contribution of this paper is therefore the development of an 
intelligent system to support online course design based solely on freely available resources on the Web.

The APELS system will address three main issues. The first is the identification of the learner's requirements, the second is the identification of the learner's learning style and the third is the ability of the APELS system to adapt and modify the content and learning style based on the interactions of the users with the system over a period of time. In addition, the information extracted by the system will be passed to a Planner module that will structure it into lectures/tutorials and workshops based on some predefined learning times. The evaluation of the learning process will be against a set of learning outcomes as defined by standard curricula. In this paper, a case study using the IEEE/ACM Computing Curriculum [11] will be used to illustrate the functionality of the APELS system.

\section{Background and Related work}

Over the last few years, researchers have started taking a keen interest in developing personalised learning systems, whose main purpose is to provide content, learning style and environments for the needs of specific learners. First, it is crucial to determine the learners' profile such as their background, level and needs, which can be determined by using a questioner. An example of such system is SPERO [1], a personalised E-learning system based on the IEEE Learning Technology Systems Architecture. The purpose of SPERO is to help teacher training in special education, which could provide different contents for foreign languages learners according to their interests and levels. However, SPERO is largely using questionnaires and e-surveys to build user profiles, which adds extra work to the users and is time consuming. Moreover, adaptive systems can be made more flexible by examining the learners' progress to make changes according to their needs. For instance, Baylari et al. [2] presented a personalized multi-agents E-Learning system based on Item Response Theory, which presents adaptive tests to estimate learners' knowledge and enables it to make changes according to their needs and background. Zhuge and Yanyan [4] proposed the KGTutor; a Knowledge grid based intelligent system personalised E-Learning system that examines each learner requirements such as previous knowledge and the learners' targets and then provides them with a personalised course. The system can also provide the learner with progress reports, evaluations and suggestions based on their performance. Ontologies are also becoming a great tool commonly used by researchers to extract educational information with the aim of assisting students to learn about specific topics. Cassin et al. [8] used the notion of ontology extraction for educational knowledge, aiming at helping students who need to learn about some specific topics.

In our work, learners' levels, background and needs will be addressed by questioners, before starting any module and their progress will be tested after each level and they will not be able to proceed until they have passed an assessment. Furthermore, this system will have an ontology structure to easily 
extract the knowledge in domain area on the WWW in order to improve information retrieval, organize and update learning resources specific to the user. In E-learning, ontologies can endorse adaptive learning services to help in the online learning process. Yarandi et al. [7] proposed a novel approach for developing personalised E-learning systems for individual learners using an ontology to create a user model to recognize learners and describe their behaviours. Moreover, they developed the learning content using an ontology to build the hierarchical and navigational relations between different parts of learning materials and how these can be determined based on users profiles.

\section{System Architecture}

The purpose of the APELS is to deliver recommended learning materials to learners who may have different backgrounds, learning styles and learning needs. The architecture is based on four main modules that will form the basis of the system. These include: student profile, student requirement, knowledge extraction system, and content delivery module as shown in Figure 1.

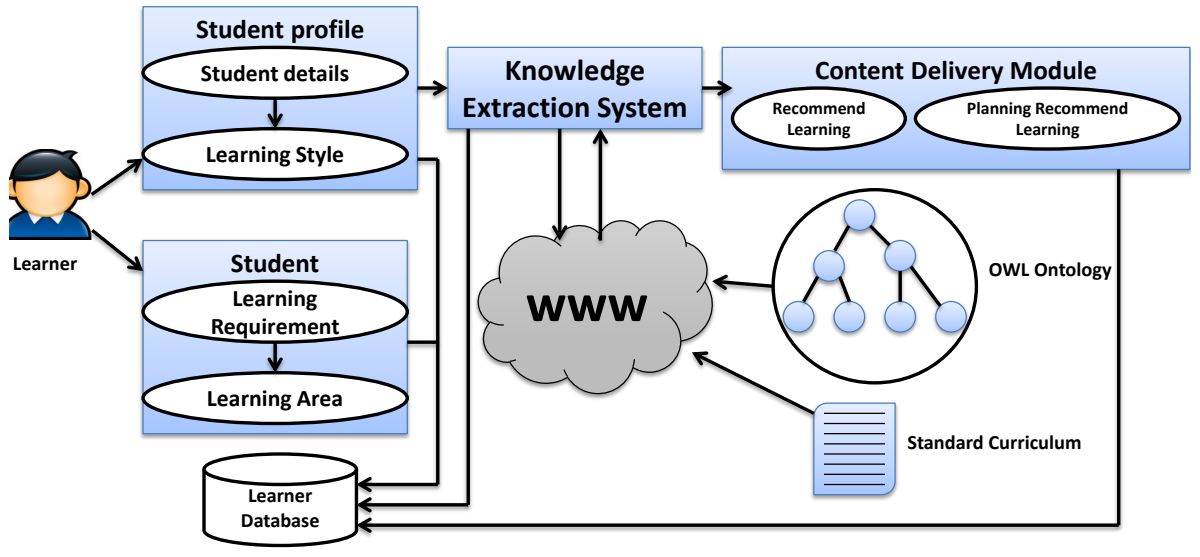

Fig. 1. System Architecture

\subsection{Student Profile}

This module contains two components; Student details and Learning style:

- Student details: This component stores the student's personal information.

- Learning Style: This component identifies the learning style of the learner using the VARK learning style [9]. 


\subsection{Student Requirements}

This module contains two components; Learning requirements and Learning area:

- Learning Requirement: This component gathers information related to the learners background and previous knowledge.

- Learning Area: In this component, the learner will choose the the subject he/she wishes to study.

\subsection{The Knowledge Extraction System}

Once the details of the learner and his chosen area are known, the information extraction module is used to extract learning resources from the Web that are suitable for the learner. This Subsystem has the following 5 components: (i) Ontology, (ii) HTML2XML, (iii) Concepts Extraction, (iv) Value Extraction, (v) Matching Process.

The Ontology The APELS system uses an ontology to help in extracting the required domain knowledge from the Web in order to improve the information retrieval process, organize and update learning resources specific to the user. To develop the ontology we used Protégé 2000 [3]. The ontology contains the standard is-a hierarchy of relevant domain concepts and relations between these concepts. Moreover, we exported the Web Ontology Language (OWL) which can represent domain knowledge using classes (concepts), properties, axioms and instances to support efficient reasoning and expressive power [12]. We will focus on concepts and relationships between them when building the Ontology. Avoiding the construction of a very large ontology that could be difficult to develop and use, we have adopted the development of smaller and specific ones that will support individual areas such as Computer Science, Law etc.

HTML2XML Most Web content is written in HTML documents and it is difficult to extract the information from HTML. Therefore we have developed a tool, HTML2XML which will automatically create XML data sources from the collected HTML Web pages. Different approaches have been used for representing Web information $[5,10]$. The traditional XML file usually represents all HTML data. We have improved this process by only selecting pertinent information.

Concepts Extraction The OWL file obtained from the Protégé tool is used to extract the concepts that are represented in a specific domain through the domain ontology. These entities are used to determine similarities with the XML files produced from HTML files. This was a very simple and straight forward process. A vector is used and populated with the ontology concepts. However, given the size of the ontology, in many operations involved in this project, we only deal with a subset of the ontology that models the learning area. 
Values Extraction In a similar way, when constructing XML files from HTML files, XML files contain element and attribute values. These are extracted and constitute the XML value vector and denoted as $V=\left[V_{1}, V_{2}, \ldots, V_{m}\right]$.

The Matching Process The matching process involves the computation of a simple similarity measure between the subset of the ontology that models the learning domain $D$ and the vector $V$ for a website $W$. Given a set of relevant websites $W S$ and their associated value vectors, the website with the highest similarity is selected as the best matching website for the learner satisfying his/her learning style. However, we do acknowledge the limitations of the current approach when running our experiments. Sometimes parts of the website only are relevant and appropriate for the learner and a combination of two or more websites contents will provide a better content. In addition, some concepts or terms may be given different names, although they have the same meaning. For instance, the equivalent terms for the concept "Calculus" include arithmetic, mathematics etc. This issue was solved by defining corresponding relations such as synonyms in the domain ontology. The similarity is calculated as follows: Given two vectors $C$ and $V$ defined as $C=\left[C_{1}, C_{2}, \ldots, C_{m}\right]$ where $C_{i}$ represent an ontology Concept and $V=\left[V_{1}, V_{2}, \ldots, V_{m}\right]$ where $V_{j}$ represent XML values. The similarity measure between vectors $\mathrm{C}$ and $\mathrm{V}$ is calculated as: $S C(C, V)=\frac{s c}{n} * 100$, where $S C$ is the number concepts in $C$ that are also present in $V$ and $n$ is the number of concepts in the subset of the ontology concepts dealing with the specific learning area of the learner.

\section{Case Study and Evaluation}

\subsection{Description of the Case Study}

To illustrate the functionality of the APELS system, we used some elements of the ACM/IEEE Computer Society Computer Science Curriculum, an internationally recognised and adopted standard in designing computer science related programs [11]. The IEEE/ACM Body of Knowledge (BoK) is organized into a set of 18 Knowledge Areas (KAs) corresponding to typical areas of study in computing such as Algorithms and Complexity, Operating Systems and Software Engineering. Each Knowledge Area (KA) is broken down into Knowledge Units (KUs). Each KU is divided into set of topics which are then classified into a tiered set of core topics (compulsory topics that must be taught) and elective topics (significant depth in many of the Elective topics should be covered). Core topics are further divided into Core-Tier1 topics and Core-Tier2 topics (Should almost be covered). The software development fundamentals area for example is divided into $4 \mathrm{KUs}$. The Algorithm and design $\mathrm{KU}$ is divided into 11 CoreTier1 topics. Learning outcomes are then defined for each class of topics. We will specifically look at designing fundamental Programming Concepts in $\mathrm{C}++$ module using the APELS system. 


\subsection{Use of the APELS System to Develop SE Learning Material}

The learner will go through the various stages as described in sections 3.1 and 3.2 and will provide the required information. He will then choose the topic to study, here computer Science/Programming. Once Programming is chosen, the list of the $\mathrm{KU}$ will be displayed for the learner to choose from. Here for clarity we attempt to design a $\mathrm{C}++$ programming module for beginners. Once a $\mathrm{KU}$ is chosen, APELS will start looking for material on the Web that will satisfy this specific learner and starts extracting the right material and organising it into smaller learning topics. The topics will then be mapped to hourly learning schedules.

\subsection{Results and Evaluation}

The launch of the APELS system will in a first instance return a list of websites for learning $\mathrm{C}++$ language with the highest accuracy rating as shown in the Table 1 that identifies that the 4 th website is the one that is most similar to the computer science Ontology represented as an OWL file. Together with the first website, they have better performances than the other websites as they have the highest similarities. The other websites show poor performance as the similarities with the required topics is low.

Table 1. Similarity Matching with the OWL file

\begin{tabular}{|l|c|c|c|}
\hline WWW & $\begin{array}{c}\text { Concepts } \\
\text { Extraction }\end{array}$ & $\begin{array}{c}\text { No of } \\
\text { Similarities }\end{array}$ & Accuracy \\
\hline www.cplusplus.com/doc/tutorial/ & 9 & 7 & $77.78 \%$ \\
\hline www.penguinprogrammer.co.uk/ & 9 & 5 & $55.56 \%$ \\
\hline www.tutorialspoint.com/cplusplus/ & 9 & 4 & $44.44 \%$ \\
\hline www.doc.ic.ac.uk/ wjk/C++Intro/ & 9 & 8 & $88.89 \%$ \\
\hline www.macs.hw.ac.uk/ pjbk/pathways/cpp1 & 9 & 3 & $33.33 \%$ \\
\hline www.cprogramming.com/tutorial.html & 9 & 2 & $22.22 \%$ \\
\hline
\end{tabular}

\section{Conclusion and Future Work}

The work presented in this paper is the first stages in developing the APELS system that attempts to develop course contents based on freely available resources on the Web. The approach, functionality and architecture are improvements on existing E-learning systems. The system was evaluated using the ACM/IEEE Computing curriculum. The next steps in developing this system will include the user characteristics and background and also the learning outcomes of each Knowledge unit or modules that are not yet implemented in the current version of the system. The full version of the system will then be evaluated using domain experts and students. 


\section{References}

1. Spero: Tele-informatics system for continuous collection, processing, diffusion of material for teacher training in special education. http://www.image.ntua.gr/ spero.

2. Baylari A. and Montazer Gh. Design a personalized e-learning system based on item response theory and artificial neural network approach. Expert Systems with Applications, 36(4):8013-8021, 2009.

3. Noy N. F. and Mcguinness D. L. Ontology development 101: A guide to creating your first ontology. Techinal Report KSL-01-05, Stanford Knowledge Systems Laboratory, 2000.

4. Zhuge H. and Yanyan L. Advanced Web Technologies and Applications, chapter KGTutor: a knowledge grid based intelligent tutoring system, pages 473-478. Springer Berlin Heidelberg, 2004.

5. Breuel T. M. Information extraction from html documents by structural matching. In Proceedings of the 2nd International Workshop on Web Document Analysis, 2002.

6. El-Zayat M. An assessment of e-learning in egypt through the perceptions of egyptian university students: A field work survey. In AUC 5th International Conference on E-Learning Applications, 2007.

7. Yarandi M., Tawil A., and Jahankhani H. Adaptive e-learning system using ontology. In 22nd International Workshop on Database and Expert Systems Applications, pages 511-516, 2011.

8. Cassin P., Eliot C., Lesser V., Rawlins K., and Woolf B. Ontology extraction for educational knowledge bases. In In Agent-Mediated Knowledge Management. Springer Berlin Heidelberg, 2004.

9. Honey P. and Mumford A. The Manual of Learning Styles. Peter Honey, Maidenhead, 3rd edition, 1992.

10. Song R., Liu H., Wen J. R., and Ma W. Y. Learning block importance models for web pages. In Proceedings of International $W W W$ Conference ( $W W W$-2004), 2004.

11. ACM/IEEE societies. Computer science curricula. http://www.acm.org/ education/CS2013-final-report.pdf, 2013.

12. W3C. Owl 2 web ontology language primer (second edition) w3c recommendation. http://www.w3.org/TR/ow12-primer/, December 2012. 